\title{
Multirate Simulations of String Vibrations Including Nonlinear Fret-String Interactions Using the Functional Transformation Method
}

\author{
L. Trautmann \\ Multimedia Communications and Signal Processing, University of Erlangen-Nuremberg, Cauerstrasse 7, 91058 Erlangen, Germany \\ Email: traut@lnt.de
}

Laboratory of Acoustics and Audio Signal Processing, Helsinki University of Technology, P.O. Box 3000, 02015 Espoo, Finland Email: lutz.trautmann@acoustics.hut.fi

\author{
R. Rabenstein \\ Multimedia Communications and Signal Processing, University of Erlangen-Nuremberg, Cauerstrasse 7, 91058 Erlangen, Germany \\ Email: rabe@lnt.de
}

Received 30 June 2003; Revised 14 November 2003

\begin{abstract}
The functional transformation method (FTM) is a well-established mathematical method for accurate simulations of multidimensional physical systems from various fields of science, including optics, heat and mass transfer, electrical engineering, and acoustics. This paper applies the FTM to real-time simulations of transversal vibrating strings. First, a physical model of a transversal vibrating lossy and dispersive string is derived. Afterwards, this model is solved with the FTM for two cases: the ideally linearly vibrating string and the string interacting nonlinearly with the frets. It is shown that accurate and stable simulations can be achieved with the discretization of the continuous solution at audio rate. Both simulations can also be performed with a multirate approach with only minor degradations of the simulation accuracy but with preservation of stability. This saves almost $80 \%$ of the computational cost for the simulation of a six-string guitar and therefore it is in the range of the computational cost for digital waveguide simulations.
\end{abstract}

Keywords and phrases: multidimensional system, vibrating string, partial differential equation, functional transformation, nonlinear, multirate approach.

\section{INTRODUCTION}

Digital sound synthesis methods can mainly be categorized into classical direct synthesis methods and physics-based methods [1]. The first category includes all kinds of sound processing algorithms like wavetable, granular and subtractive synthesis, as well as abstract mathematical models, like additive or frequency modulation synthesis. What is common to all these methods is that they are based on the sound to be (re)produced.

The physics-based methods, also called physical modeling methods, start at the physics of the sound production mechanism rather than at the resulting sound. This approach has several advantages over the sound-based methods.

(i) The resulting sound and especially transitions between successive notes always sound acoustically realistic as far as the underlying model is sufficiently accurate.

(ii) Sound variations of acoustical instruments due to dif- ferent playing techniques or different instruments within one instrument family are described in the physics-based methods with only a few parameters. These parameters can be adjusted in advance to simulate a distinct acoustical instrument or they can be controlled by the musician to morph between real world instruments to obtain more degrees of freedom in the expressiveness and variability.

The second item makes physical modeling methods quite useful for multimedia applications where only a very limited bandwidth is available for the transmission of music as, for example, in mobile phones. In these applications, the physical model has to be transferred only once and afterwards it is sufficient to transfer only the musical score while keeping the variability of the resulting sound.

The starting points for the various existing physical modeling methods are always physical models varying for a certain vibrating object only in the model accuracies. The application of the basic laws of physics to an existing or imaginary 
vibrating object results in continuous-time, continuousspace models. These models are called initial-boundaryvalue problems and they contain a partial differential equation (PDE) and some initial and boundary conditions. The discretization approaches to the continuous models and the digital realizations are different for the single physical modeling methods.

One of the first physical modeling algorithm for the simulation of musical instruments was made by Hiller and Ruiz 1971 in [2] with the finite difference method. It directly discretizes the temporal and spatial differential operators of the PDE to finite difference terms. On the one hand, this approach is computationally very demanding; since temporal and spatial sampling intervals have to be chosen small for accurate simulations. Furthermore, stability problems occur especially in dispersive vibrational objects if the relationship between temporal and spatial sampling intervals is not chosen properly [3]. On the other hand, the finite difference method is quite suitable for studies in which the vibration has to be evaluated in a dense spatial grid. Therefore, the finite difference method has mainly been used for academic studies rather than for real-time applications (see, e.g., $[4,5]$ ). However, the finite difference method has recently become more popular also for real-time applications in conjunction with other physical modeling methods $[6,7]$.

A mathematically similar discretization approach is used in mass-spring models that are closely related to the finite element method. In this approach, the vibrating structure is reduced to a finite number of mass points that are interconnected by springs and dampers. One of the first systems for the simulation of musical instruments was the CORDIS system which could be realized in real time on a specialized processor [8]. The finite difference method, as well as the mass-spring models, can be viewed as direct discretization approaches of the initial-boundary-value problems. Despite the stability problems, they are very easy to set up, but they are computationally demanding.

In modal synthesis, first introduced in [9], the PDE is spatially discretized at non necessarily equidistant spatial points, similar to the mass-spring models. The interconnections between these discretized spatial points reflect the physical behavior of the structure. This discretization reduces the degrees of freedom for the vibration to the number of spatial points which is directly transferred to the same number of temporal modes the structure can vibrate in. The reduction does not only allow the calculation of the modes of simple structures, but it can also handle vibrational measurements of more complicated structures at a finite number of spatial points [10]. A commercial product of the modal synthesis, Modalys, is described, for example, in [11]. For a review of modal synthesis and a comparison to the functional transformation method (FTM), see also [12].

The commercially and academically most popular physical modeling method of the last two decades was the digital waveguide method (DWG) because of its computational efficiency. It was first introduced in [13] as a physically interpreted extension of the Karplus-Strong algorithm [14]. Extensions of the DWG are described, for example, in $[15,16$,
17, 18]. The DWG first simplifies the PDE to the wave equation which has an analytical solution in the form of a forward and backward traveling wave, called d'Alembert solution. It can be realized computationally very efficient with delay lines. The sound effects like damping or dispersion occurring in the vibrating structure are included in the DWG by low-order digital filters concentrated in one point of the delay line. This procedure ensures the computational efficiency, but the implementation looses the direct connection to the physical parameters of the vibrating structure.

The focus of this article is the FTM. It was first introduced in [19] for the heat-flow equation and first used for digital sound synthesis in [20]. Extensions to the basic model of a vibrating string and comparisons between the FTM and the above mentioned physical modeling methods are given, for example, in [12]. In the FTM, the initial-boundary-value problem is first solved analytically by appropriate functional transformations before it is discretized for computer simulations. This ensures a high simulation accuracy as well as an inherent stability. One of the drawbacks of the FTM is so far its computational load, which is about five times higher than the load of the DWG [21].

This article extends the FTM by applying a multirate approach to the discrete realization of the FTM, such that the computational complexity is significantly reduced. The extension is shown for the linearly vibrating string as well as for the nonlinear limitation of the string vibration by a fretstring interaction occurring in slapbass synthesis.

The article is organized as follows. Section 2 derives the physical model of a transversal vibrating, dispersive, and lossy string in terms of a scalar PDE and initial and boundary conditions. Furthermore, a model for a nonlinear fret-string interaction is given. These models are solved in Section 3 with the FTM in continuous time and continuous space. Section 4 discretizes these solutions at audio rate and derives an algorithm to guarantee stability even for the nonlinear discrete system. A multirate approach is used in Section 5 for the simulation of the continuous solution to save computational cost. It is shown that this multirate approach also works for nonlinear systems. Section 6 compares the audio rate and the multirate solutions with respect to the simulation accuracy and the computational complexity.

\section{PHYSICAL MODELS}

In this Section, a transversal vibrating, dispersive, and lossy string is analyzed using the basic laws of physics. From this analysis, a scalar PDE is derived in Section 2.1. Section 2.2 defines the initial states of the vibration, as well as the fixings of the string at the nut and the bridge end, in terms of initial and boundary conditions, respectively. In Section 2.3, the linear model is extended with a deflection-dependent force simulating the nonlinear interaction between the string and the frets, well known as slap synthesis [22].

In all these models, the strings are assumed to be homogeneous and isotropic. Furthermore, the smoothness of their surfaces may not permit stress concentrations. The deflections of the strings are assumed to be small enough to change 
neither the cross section area nor the tension on the string so that the string itself behaves linearly.

\subsection{Linear partial differential equation derived by basic laws of physics}

The string under examination is characterized by its material and geometrical parameters. The material parameters are given by the mass density $\rho$, the Young's modulus $E$, the laminar air flow damping coefficient $d_{1}$, and the viscoelastic damping coefficient $d_{3}$. The geometrical parameters consist of the length $l$, the cross section area $A$ and the moment of inertia $I$. Furthermore, a tension $T_{\mathrm{s}}$ is applied to the string in axial direction. Considering only a string segment between the spatial positions $x_{\mathrm{s}}$ and $x_{\mathrm{s}}+\Delta x$, the forces on this string segment can be analyzed in detail. They consist of the restoring force $f_{\mathrm{T}}$ caused by the tension $T_{\mathrm{s}}$, the bending force $f_{\mathrm{B}}$ caused by the stiffness of the string, the laminar air flow force $f_{\mathrm{d} 1}$, the viscoelastic damping force $f_{\mathrm{d} 3}$ (modeled here without memory), and the external excitation force $f_{\mathrm{e}}$. They result at $x_{\mathrm{s}}$ in

$$
\begin{gathered}
f_{\mathrm{T}}\left(x_{\mathrm{s}}, t\right)=T_{\mathrm{s}} \sin \left(\varphi\left(x_{\mathrm{s}}, t\right)\right) \approx T_{\mathrm{s}} \varphi\left(x_{\mathrm{s}}, t\right), \\
f_{\mathrm{B}}\left(x_{\mathrm{s}}, t\right)=-E I b^{\prime}\left(x_{\mathrm{s}}, t\right), \\
f_{\mathrm{d} 1}\left(x_{\mathrm{s}}, t\right)=d_{1} \Delta x v\left(x_{\mathrm{s}}, t\right), \\
f_{\mathrm{d} 3}\left(x_{\mathrm{s}}, t\right)=d_{3} \sin \left(\dot{\varphi}\left(x_{\mathrm{s}}, t\right)\right) \approx d_{3} \dot{\varphi}\left(x_{\mathrm{s}}, t\right),
\end{gathered}
$$

where $\varphi\left(x_{\mathrm{s}}, t\right)$ is the slope angle of the string, $b\left(x_{\mathrm{s}}, t\right)$ is the curvature of the string, $v\left(x_{\mathrm{s}}, t\right)$ is the velocity, and prime denotes spatial derivative and dot denotes temporal derivative. Note that in (1a) and in (1d) it is assumed that the amplitude of the string vibration is small so that the sine function can be approximated by its argument. Similar equations can be found for the forces at the other end of the string segment at $x_{s}+\Delta x$.

All these forces are combined by the equation of motion to

$$
\begin{aligned}
\rho A \Delta x \dot{v}\left(x_{\mathrm{s}}, t\right)= & f_{y}\left(x_{\mathrm{s}}, t\right)+f_{\mathrm{d} 3}\left(x_{\mathrm{s}}, t\right)-f_{y}\left(x_{\mathrm{s}}+\Delta x, t\right) \\
& -f_{\mathrm{d} 3}\left(x_{\mathrm{s}}+\Delta x, t\right)-f_{\mathrm{d} 1}\left(x_{\mathrm{s}}, t\right)+f_{\mathrm{e}}\left(x_{\mathrm{s}}, t\right),
\end{aligned}
$$

where $f_{y}=f_{\mathrm{T}}+f_{\mathrm{B}}$. Setting $\Delta x \rightarrow 0$ and solving (2) for the excitation force density $f_{\mathrm{e} 1}\left(x_{\mathrm{s}}, t\right)=f_{\mathrm{e}}\left(x_{\mathrm{s}}, t\right) \delta\left(x-x_{\mathrm{s}}\right)$, four coupled equations are obtained, that are valid not only at the string segment $x_{\mathrm{s}} \leq x \leq x_{\mathrm{s}}+\Delta x$ but also at the whole string $0 \leq x \leq l . \delta(x)$ denotes the impulse function.

$$
\begin{gathered}
f_{\mathrm{el}}(x, t)=\rho A \dot{v}(x, t)+d_{1} v(x, t)-f_{y}^{\prime}(x, t)-d_{3} \dot{b}(x, t) \\
f_{y}(x, t)=T_{s} \varphi(x, t)-E I b^{\prime}(x, t) \\
b\left(x_{1}, t\right)=\varphi^{\prime}(x, t) \\
v^{\prime}\left(x_{1}, t\right)=\dot{\varphi}(x, t) .
\end{gathered}
$$

An extended version of the derivation of (3) can be found in [12]. The four coupled equations (3) can be simplified to one scalar PDE with only one output variable. All the dependent variables in (3a) can be written in terms of the string deflection $y(x, t)$ by replacing $v(x, t)$ with $\dot{y}(x, t)$ and $\varphi(x, t)=y^{\prime}(x, t)$ from (3d) and with (3b) and (3c). Then (3) can be written in a general notation of scalar PDEs

$$
\begin{aligned}
\mathrm{D}\{y(x, t)\}+\mathrm{L}\{y(x, t)\} & +\mathrm{W}\{y(x, t)\} \\
= & f_{\mathrm{el}}(x, t), \quad x \in[0, l], t \in[0, \infty),
\end{aligned}
$$

with

$$
\begin{aligned}
\mathrm{D}\{y(x, t)\} & =\rho A \ddot{y}(x, t)+d_{1} \dot{y}(x, t), \\
\mathrm{L}\{y(x, t)\} & =-T_{\mathrm{s}} y^{\prime \prime}(x, t)+E I_{\mathrm{B}} y^{\prime \prime \prime \prime}(x, t), \\
\mathrm{W}\{y(x, t)\} & =\mathrm{W}_{\mathrm{D}}\left\{\mathrm{W}_{\mathrm{L}}\{y(x, t)\}\right\}=-d_{3} \dot{y}^{\prime \prime}(x, t) .
\end{aligned}
$$

As it can be seen in (4), the operator $\mathrm{D}\{\}$ contains only temporal derivatives, the operator $\mathrm{L}\{\}$ has only spatial derivatives, and the operator $\mathrm{W}\{\}$ consists of mixed temporal and spatial derivatives. The PDE is valid only on the string between $x=0$ and $x=l$ and for all positive times. Equation (4) forms a continuous-time, continuous-space PDE. For a unique solution, initial and boundary conditions must be given as specified in the next section.

\subsection{Initial and boundary conditions}

Initial conditions define the initial state of the string at time $t=0$. This definition is written in the general operator notation with

$$
\mathbf{f}_{\mathrm{i}}^{T}\{y(x, t)\}=\left[\begin{array}{c}
y(x, 0) \\
\dot{y}(x, 0)
\end{array}\right]=\mathbf{0}, \quad x \in[0, l], t=0 .
$$

Since the scalar PDE (4) is of second order with respect to time, only two initial conditions are needed. They are chosen arbitrarily by the initial deflection and the initial velocity of the string as seen in (5). For musical applications, it is a reasonable assumption that the initial states of the strings vanish at time $t=0$ as given in (5). Note that this does not prevent the interaction between successively played notes since the time is not set to zero for each note. Thus, this kind of initial condition is only used for, for example, the beginning of a piece of music.

In addition to the initial conditions, also the fixings of the string at both ends must be defined in terms of boundary conditions. In most stringed instruments, the strings are nearly fixed at the nut end $\left(x=x_{0}=0\right)$ and transfer energy at the other end $\left(x=x_{1}=l\right)$ via the bridge to the resonant body [2]. For some instruments (e.g., the piano) it is also a justified assumption, that the bridge fixing can be modeled to be ideally rigid [23]. Then the boundary conditions are given by

$$
\mathbf{f}_{\mathrm{b} i}^{T}\{y(x, t)\}=\left[\begin{array}{c}
y\left(x_{i}, t\right) \\
y^{\prime \prime}\left(x_{i}, t\right)
\end{array}\right]=\mathbf{0}, \quad i \in 0,1, t \in[0, \infty) .
$$

It can be seen from (6) that the string is assumed to be fixed, allowed to pivot at both ends, such that the deflection $y$ and the curvature $b=y^{\prime \prime}$ must vanish. These are boundary conditions of first kind. For simplicity, there is no energy fed 


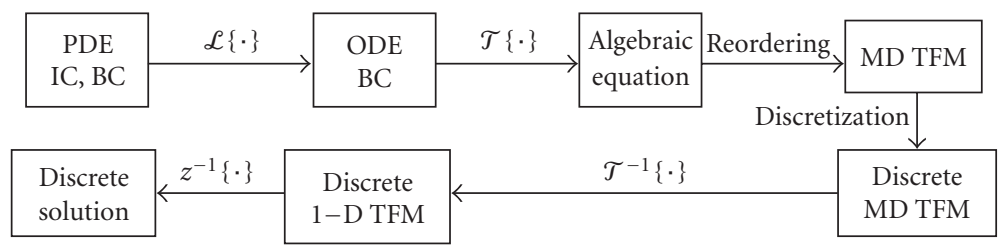

FIgURE 1: Procedure of the FTM solving initial boundary value problems defined in form of PDEs, IC, and BC.

into the system via the boundary, resulting in homogeneous boundary conditions.

The PDE (4), in conjunction with the initial (5) and boundary conditions (6), forms the linear continuoustime continuous-space initial-boundary-value problem to be solved and simulated.

\subsection{Nonlinear extension to the linear model for slap synthesis}

Nonlinearities are an important part in the sound production mechanisms of musical instruments [23]. One example is the nonlinear interaction of the string with the frets, well known as slap synthesis. This effect was modeled first for the DWG in [22] as a nonlinear amplitude limitation. For the FTM, the effect was already applied to vibrating strings in [24].

A simplified model for this interaction interprets the fret as a spring with a high stiffness coefficient $S_{\text {fret }}$ acting at one position $x_{\mathrm{f}}$ as a force $f_{\mathrm{f}}$ on the string at time instances where the string is in contact with the fret. Since this force depends on the string deflection, it is nonlinear, defined with

$$
\begin{aligned}
& f_{\mathrm{f}}\left(x_{\mathrm{f}}, t, y, y_{\mathrm{f}}\right) \\
& \quad= \begin{cases}S_{\mathrm{fret}}\left(y\left(x_{\mathrm{f}}, t\right)-y_{\mathrm{f}}\left(x_{\mathrm{f}}, t\right)\right), & \text { for } y\left(x_{\mathrm{f}}, t\right)-y_{\mathrm{f}}\left(x_{\mathrm{f}}, t\right)>0, \\
0, & \text { for } y\left(x_{\mathrm{f}}, t\right)-y_{\mathrm{f}}\left(x_{\mathrm{f}}, t\right) \leq 0 .\end{cases}
\end{aligned}
$$

The deflection of the fret from the string rest position is denoted with $y_{\mathrm{f}}$. The PDE (4) becomes nonlinear by adding the slap force $f_{\mathrm{f}}$ to the excitation function $f_{\mathrm{el}}(x, t)$. Thus, a linear and a nonlinear system for the simulation of the vibrating string is derived. Both systems are solved in the next sections with the FTM.

\section{CONTINUOUS SOLUTIONS USING THE FTM}

To obtain a model that can be implemented in the computer, the continuous initial-boundary-value problem has to be discretized. Instead of using a direct discretization approach as described in Section 1, the continuous analytical solution is derived first, which is discretized subsequently. This procedure is well known from the simulation of one-dimensional systems like electrical networks. It has several advantages including simulation accuracy and guaranteed stability.

The outline of the FTM is given in Figure 1. First, the PDE with initial conditions (IC) and boundary conditions
(BC) is Laplace transformed $(\mathcal{L}\{\cdot\})$ with respect to time to derive a boundary-value problem (ODE, BC). Then a so-called Sturm-Liouville transformation $(\mathcal{T}\{\cdot\})$ is used for the spatial variable to obtain an algebraic equation. Solving for the output variable results in a multidimensional (MD) transfer function model (TFM). It is discretized and by applying the inverse Sturm-Liouville transformation $\mathcal{T}^{-1}\{\cdot\}$ and the inverse $z$-transformation $z^{-1}\{\cdot\}$ it results in the discretized solution in the time and space domain.

The impulse-invariant transformation is used for the discretization shown in Figure 1. It is equivalent to the calculation of the continuous solution by inverse transformation into the continuous time and space domain with subsequent sampling. The calculation of the continuous solution is presented in Sections 3.1 to 3.5 , the discretization is shown in Sections 4 and 5.

For the nonlinear system, the transformations cannot obviously result in a TFM. Therefore, the procedure has to be modified slightly, resulting in an MD implicit equation, described in Section 3.6.

\subsection{Laplace transformation}

As known from linear electrical network theory, the Laplace transformation removes the temporal derivatives in linear and time-invariant (LTI) systems and includes, due to the differentiation theorem, the initial conditions as additive terms (see, e.g., [25]). Since first- and second-order time derivatives occur in (4) and the initial conditions (5) are homogeneous, the application of the Laplace transformation to the initial boundary value problem derived in Section 2 results in

$$
\begin{gathered}
d_{\mathrm{D}}(s) Y(x, s)+\mathrm{L}\{Y(x, s)\}+w_{\mathrm{D}}(s) \mathrm{W}_{\mathrm{L}}\{Y(x, s)\} \\
=F_{\mathrm{e} 1}(x, s), \quad x \in[0, l], \\
\mathbf{f}_{\mathrm{b} i}^{T} \mathbf{Y}(x, s)=\mathbf{0}, \quad i \in 0,1 .
\end{gathered}
$$

The Laplace transformed functions are written with capital letters and the complex temporal frequency variable is denoted by $s=\sigma+j \omega$. It can be seen in (8a) that the temporal derivatives of $(4 \mathrm{a})$ are replaced with scalar multiplication of the functions

$$
d_{\mathrm{D}}(s)=\rho A s^{2}+d_{1} s, \quad w_{\mathrm{D}}(s)=-d_{3} s .
$$

Thus, the initial boundary value problem (4), (5), and (6) is replaced with the boundary-value problem (8) after Laplace transformation. 


\subsection{Sturm-Liouville transformation}

The transformation of the spatial variable should have the same properties as the Laplace transformation has for the time variable. It should remove the spatial derivatives and it should include the boundary conditions as additive terms. Unfortunately, there is no unique transformation available for this task due to the finite spatial definition range in contrast to the infinite time axis. That calls for a determination of the spatial transformation at hand, depending on the spatial differential operator and the boundary conditions. Since it leads to an eigenvalue problem first solved for simplified problems by Sturm and Liouville between 1836 and 1838, this transformation is called a Sturm-Liouville transformation (SLT) [26]. Mathematical details of the SLT applied to scalar PDEs can be found in [12].

The SLT is defined by

$$
\mathcal{T}\{Y(x, s)\}=\bar{Y}(\mu, s)=\int_{0}^{l} K(\mu, x) Y(x, s) \mathrm{d} x .
$$

Note that there is a finite integration range in (9) in contrast to the Laplace transformation. The transformation kernels $K(\mu, x)$ of the SLT are obtained as the set of eigenfunctions of the spatial operator $\mathrm{L}_{\mathrm{W}}=\mathrm{L}+\mathrm{W}_{\mathrm{L}}$ with respect to the boundary conditions $(8 \mathrm{~b})$. The corresponding eigenvalues are denoted by $\beta_{\mu}^{4}(s)$ where $\beta_{\mu}(s)$ is the discrete spatial frequency variable (see, e.g., [12] for details).

For the boundary-value problem defined in (8) with the operators given in $(4 \mathrm{~b})$, the transformation kernels and the discrete spatial frequency variables result in

$$
\begin{aligned}
K(\mu, x) & =\sin \left(\frac{\mu \pi}{l} x\right), \quad \mu \in \mathbb{N} \\
\beta_{\mu}^{4}(s) & =E I\left(\frac{\mu \pi}{l}\right)^{4}-\left(T_{\mathrm{s}}+d_{3} s\right)\left(\frac{\mu \pi}{l}\right)^{2} .
\end{aligned}
$$

Thus, the SLT can be interpreted as an extended Fourier series decomposition.

\subsection{Multidimensional transfer function model}

Applying the SLT (9) to the boundary-value problem (8) and solving for the transformed output variable $\bar{Y}(\mu, s)$ results in the MD TFM

$$
\bar{Y}(\mu, s)=\frac{1}{d_{\mathrm{D}}(s)+\beta_{\mu}^{4}(s)} \bar{F}_{\mathrm{e}}(\mu, s) .
$$

Hence, the transformed input forces $\bar{F}(\mu, s)$ are related via the MD transfer function given in (11) to the transformed output variable $\bar{Y}(\mu, s)$. The denominator of the MD TFM depends quadratically on the temporal frequency variable $s$ and to the power of four on the spatial frequency variable $\beta_{\mu}$. This is based on the second-order temporal and fourth-order spatial derivatives occurring in the scalar PDE (4). Thus, the transfer function is a two-pole system with respect to time for each discrete spatial eigenvalue $\beta_{\mu}$.

\subsection{Inverse transformations}

As explained at the beginning of Section 3, the continuous solution in the time and space domain is now calculated by using inverse transformations.

\section{Inverse SLT}

The inverse SLT is defined by an infinite sum over all discrete eigenvalues $\beta_{\mu}$ with

$$
Y(x, s)=\mathcal{T}^{-1}\{\bar{Y}(\mu, s)\}=\sum_{\mu} \frac{1}{N_{\mu}} \bar{Y}(\mu, s) K(\mu, x) .
$$

The inverse transformation kernel $K(\mu, x)$ and the inverse spatial frequency variable $\beta_{\mu}$ are the same eigenfunctions and eigenvalues as for the forward transformation due to the selfadjointness of the spatial operators $L$ and $W_{L}$ (see [12] for details). Thus, the inverse SLT can be evaluated at each spatial position by evaluating the infinite sum. Since only quadratic terms of $\mu$ occur in the denominator, it is sufficient to sum over positive values of $\mu$ and double the result to account for the negative values. The norm factor results in that case in $N_{\mu}=l / 4$.

\section{Inverse Laplace transformation}

It can be seen from (11) and (8c), (10b) that the transfer functions consist of two-pole systems with conjugate complex pole pairs for each discrete spatial eigenvalue $\beta_{\mu}$. Therefore the inverse Laplace transformation results for each spatial frequency variable in a damped sinusoidal term, called mode.

\subsection{Continuous solution}

After applying the inverse transformations to the MD TFM, the continuous solution results in

$y(x, t)=\frac{4}{\rho A l} \sum_{\mu=1}^{\infty}\left(\frac{1}{\omega_{\mu}} e^{\sigma_{\mu} t} \sin \left(\omega_{\mu} t\right) * \bar{f}_{\mathrm{e}}(x, t)\right) K(\mu, x) \delta_{-1}(t)$.

The step function, denoted by $\delta_{-1}(t)$, is used since the solution is only valid for positive time instances; $*$ means temporal convolution. $\bar{f}_{\mathrm{e}}(x, t)$ is the spatially transformed excitation force, derived by inserting $f_{\mathrm{e}}$ into (9). The angular frequencies $\omega_{\mu}$, as well as their corresponding damping coefficients $\sigma_{\mu}$, can be calculated from the poles of the transfer function model (11). They directly depend on the physical parameters of the string and can be expressed by

$$
\begin{aligned}
& \omega_{\mu}= \\
& \sqrt{\left(\frac{E I}{\rho A}-\left(\frac{d_{3}}{2 \rho A}\right)^{2}\right)\left(\frac{\mu \pi}{l}\right)^{4}+\left(\frac{T_{s}}{\rho A}-\frac{d_{1} d_{3}}{2(\rho A)^{2}}\right)\left(\frac{\mu \pi}{l}\right)^{2}-\left(\frac{d_{1}}{2 \rho A}\right)^{2}}, \\
& \sigma_{\mu}=-\frac{d_{1}}{2 \rho A}-\frac{d_{3}}{2 \rho A}\left(\frac{\mu \pi}{l}\right)^{2} .
\end{aligned}
$$

Thus, an analytical continuous solution (13), (14) of the initial boundary value problem (4), (5), (6) is derived without temporal or spatial derivatives. 


\subsection{Implicit equation for slap synthesis}

The PDE (4) becomes nonlinear by adding the solutiondependent slap force $f_{\mathrm{f}}\left(x_{\mathrm{f}}, t, y, y_{\mathrm{f}}\right)$ in (7) to the right-hand side of the linear PDE. Obviously, the application of the Laplace transformation and the SLT to the nonlinear initialboundary-value problem cannot lead to an MD TFM, since a TFM always requires linearity. However, assuming that the nonlinearity can be represented as a finite power series and that the nonlinearity does not contain spatial derivatives, both transformations can be applied to the system [12]. With (7), both premises are given such that the slap force can also be transformed into the frequency domains. The $Y(x, s)$ dependency of $\bar{F}_{\mathrm{f}}$ can be expressed with (12) in terms of $\bar{Y}(\nu, s)$ to be consistently in the spatial frequency domain. Then an MD implicit equation is derived in the temporal and spatial frequency domain

$$
\bar{Y}(\mu, s)=\frac{1}{d_{\mathrm{D}}(s)+\beta_{\mu}^{4}(s)}\left(\bar{F}_{\mathrm{e}}(\mu, s)+\bar{F}_{\mathrm{f}}(\mu, s, \bar{Y}(\nu, s))\right) .
$$

Note that the different argument $v$ in the output dependence of $\bar{F}_{\mathrm{f}}(\mu, s, \bar{Y}(\nu, s))$ denotes an interaction between all modes caused by the nonlinear slap force. Details can be found in [12].

Since the transfer functions in (11) and (15) are the same, also the spatial transformation kernels and frequency variables stay the same as in the linear case. Thus, also the temporal poles of (15) are the same as in the MD TFM (11) and the continuous solution results in the implicit equation

$$
\begin{aligned}
y(x, t)=\frac{4}{\rho A l} \sum_{\mu=1}^{\infty} & \left(\frac{1}{\omega_{\mu}} e^{\sigma_{\mu} t} \sin \left(\omega_{\mu} t\right)\right. \\
& \left.*\left(\bar{f}_{\mathrm{e}}(x, t)+\bar{f}_{\mathrm{f}}(\mu, t, \bar{y}(\nu, t))\right)\right) \\
& \times K(\mu, x) \delta_{-1}(t)
\end{aligned}
$$

with $\omega_{\mu}$ and $\sigma_{\mu}$ given in (14). It is shown in the next sections that this implicit equation is turned into explicit ones by applying different discretization schemes.

\section{DISCRETIZATION AT AUDIO RATE}

This section describes the discretization of the continuous solutions for the linear and the nonlinear cases. It is performed at audio rate, for example with sampling frequency $f_{\mathrm{s}}=1 / T=44.1 \mathrm{kHz}$, where $T$ denotes the sampling interval. The discrete realization is shown as it can be implemented in the computer. For the nonlinear slap synthesis, some extensions of the discrete realization are required and, furthermore, the stability of the entire system must be controlled.

\subsection{Discretization of the linear MD model}

The discrete realization of the MD TFM (11) consists of a three-step procedure performed below:

(1) discretization with respect to time,

(2) discretization with respect to space,

(3) inverse transformations.

\section{Discretization with respect to time}

Discretizing the time variable with $t=k T, k \in \mathbb{N}$ and assuming an impulse-invariant system, an $s$-to- $z$ mapping is applied to the MD TFM (11) with $z=e^{-s T}$. This procedure directly leads to an MD TFM with the discrete-time frequency variable $z$ :

$$
\bar{Y}^{\mathrm{d}}(\mu, z)=\frac{T\left(1 / \rho A \omega_{\mu}\right) z e^{\sigma_{\mu} T} \sin \left(\omega_{\mu} T\right)}{z^{2}-2 z e^{\sigma_{\mu} T} \cos \left(\omega_{\mu} T\right)+e^{2 \sigma_{\mu} T}} \bar{F}_{\mathrm{e}}^{\mathrm{d}}(\mu, z) .
$$

Superscript d denotes discretized variables. The angular frequency variables and the damping coefficients are given in (14). Pole-zero diagrams of the continuous and the discrete system are shown in [27].

\section{Discretization with respect to space}

For the spatial frequency domain, there is no need for discretization, since the spatial frequency variable is already discrete. However, a discretization has to be applied to the spatial variable $x$. This spatial discretization consists of simply evaluating the analytical solution (13) at a limited number of arbitrary spatial positions $x_{a}$ on the string. They can be chosen to be the pickup positions or the fret positions, respectively.

\section{Inverse transformations}

The inverse SLT cannot be performed any longer for an infinite number of $\mu$ due to the temporal discretization. To avoid temporal aliasing the number must be limited to $\mu_{T}$ such that $\left|\omega_{\mu_{T}} T\right| \leq \pi$, which also ensures realizable computer implementations. Effects of this truncation are described in [12]. The most important conclusion is that the sound quality is not effected since only modes beyond the audible range are neglected.

By applying the shifting theorem, the inverse $z$-transformation results in $\mu_{T}$ second-order recursive systems in parallel, each one realizing one vibrational mode of the string. The structure is shown with solid lines in Figure 2.

This linear structure can be implemented directly in the computer since it only includes delay elements $z^{-1}$, adders, and multipliers. Due to (14), the coefficients of the secondorder recursive systems in Figure 2 only depend on the physical parameters of the vibrating string.

\subsection{Extensions for slap synthesis}

The discretization procedure for the nonlinear slap synthesis can be performed with the same three steps described in Section 4.1. Here, the discretized MD TFM is extended with the output-dependent slap force $\bar{F}_{\mathrm{f}}^{\mathrm{d}}\left(\mu, z, \bar{Y}^{\mathrm{d}}(\nu, z)\right)$ and thus stays implicit. However, after discretization with respect to space as described above, and inverse $z$-transformation with application of the shifting theorem, the resulting recursive systems are explicit. This is caused by the time shift of the excitation function due to the multiplication with $z$ in the numerator of (17). Therefore, the linear system given with solid lines in Figure 2 is extended with feedback paths denoted by dashed lines from the output to additional inputs between 


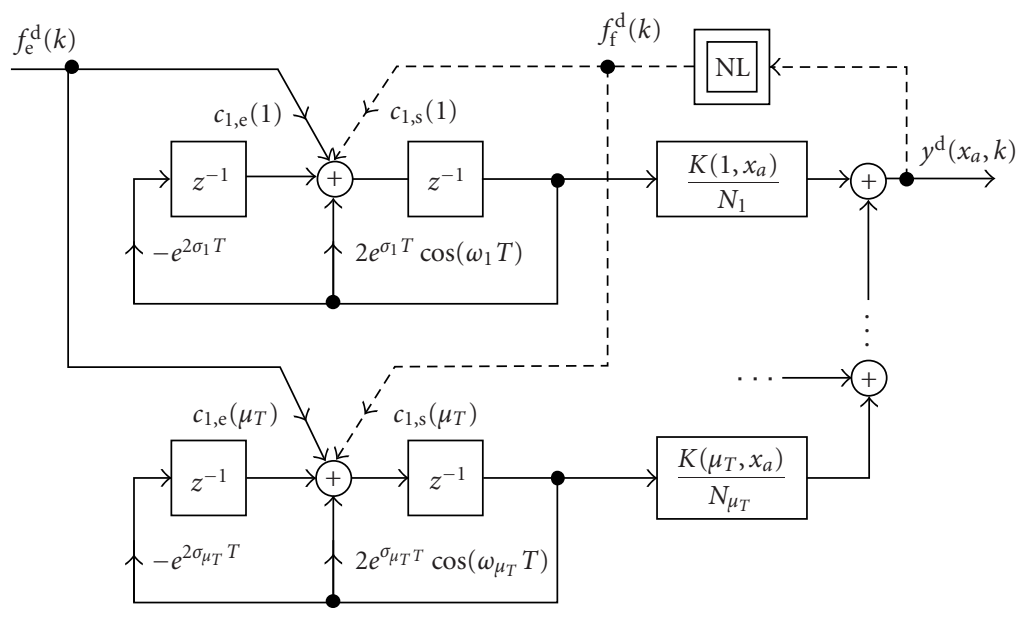

FIgURE 2: Basic structure of the FTM simulations derived from the linear initial boundary value problem (4), (5), and (6) with several second-order resonators in parallel. Solid lines represent basic linear system, while dashed lines represent extensions for the nonlinear slap force.

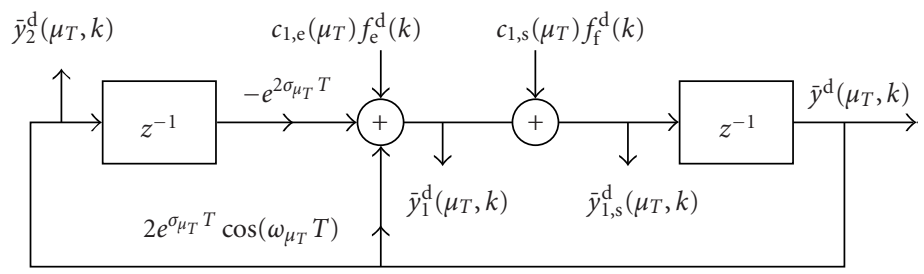

FIgURE 3: Recursive system realization of one mode of the transversal vibrating string.

the unit delays of all recursive systems. The feedback paths are weighted with the nonlinear (NL) function (7).

\subsection{Guaranteeing stability}

The discretized LTI systems derived in Section 4.1 are inherently stable as long as the underlying continuous physical model is stable due to the use of the impulse-invariant transformation [25]. However, for the nonlinear system derived in Section 4.2 this stability consideration is not valid any more. It might happen that the passive slap force of the continuous system becomes active with the direct discretization approach [24]. To preserve the passivity of the system, and thus the inherent stability, the slap force must be limited such that the discrete impulses correspond to their continuous counterparts.

The instantaneous energy of the string vibration can be calculated by monitoring the internal states of the modal deflections [12]. The slap force limitation can then be obtained directly from the available internal states. For an illustration of these internal states, the recursive system of one mode $\mu_{T}$ is given in Figure 3.

The variables $c_{1, \mathrm{e}}\left(\mu_{T}\right)$ and $c_{1, \mathrm{~s}}\left(\mu_{T}\right)$, denoting the weightings of the linear excitation force $f_{\mathrm{e}}^{\mathrm{d}}(k)$ at $x_{\mathrm{e}}$ and of the slap force $f_{\mathrm{f}}^{\mathrm{d}}(k)$ at $x_{\mathrm{f}}$, respectively, result with (9), (10a) and (17) in

$$
c_{1,(\mathrm{e}, \mathrm{s})}\left(\mu_{T}\right)=\frac{2 T}{\rho A \omega_{\mu_{T}}} \sin \left(\omega_{\mu_{T}} T\right) \sin \left(\frac{\mu_{T} \pi}{l} x_{(\mathrm{e}, \mathrm{s})}\right) .
$$

The total instantaneous energy of the string vibration without slap force density can be calculated with $[12,28]$ (time step $k$ and mode number $\mu_{T}$ dependencies are omitted for concise notation)

$$
\begin{aligned}
E_{\text {vibr }}(k)= & \frac{4 \rho A}{l} \sum_{\mu_{T}}\left(\sigma_{\mu_{T}}^{2}+\omega_{\mu_{T}}^{2}\right) \\
& \times \frac{\bar{y}_{1}^{\mathrm{d} 2}-2 \bar{y}_{1}^{\mathrm{d}} \bar{y}_{2}^{\mathrm{d}} e^{\sigma_{\mu_{T}} T} \cos \left(\omega_{\mu_{T}} T\right)-\bar{y}_{2}^{\mathrm{d} 2} e^{2 \sigma_{\mu_{T}} T}}{e^{2 \sigma_{\mu_{T}} T} \sin ^{2}\left(\omega_{\mu_{T}} T\right)} .
\end{aligned}
$$

In (19), the instantaneous energy is calculated without application of the slap force since the internal states $\bar{y}_{1}^{\mathrm{d}}\left(\mu_{T}, k\right)$ are used (see Figure 3). For calculating the instantaneous energy $E_{\mathrm{s}}(k)$ after applying the slap force, $\bar{y}_{1}^{\mathrm{d}}\left(\mu_{T}, k\right)$ must be replaced with $\bar{y}_{1, \mathrm{~s}}^{\mathrm{d}}\left(\mu_{T}, k\right)$ in (19). To meet the condition of passivity of the elastic slap collision, both energies must be related by $E_{\text {vibr }}(k) \geq E_{\mathrm{s}}(k)$. Here, only the worst-case scenario with regard to the instability problem is discussed, where both 
energies are the same. By inserting into this energy equality the corresponding expressions of (19) and solving for the slap force $f_{\mathrm{f}}^{\mathrm{d}}(k)$ results in

$$
\begin{aligned}
& f_{\mathrm{f}}^{\mathrm{d}}(k) \\
& =\sum_{\mu_{T}} c_{5}\left(\mu_{T}\right)\left(2 e^{\sigma_{\mu_{T}} T} \cos \left(\omega_{\mu_{T}} T\right) \bar{y}_{2}^{\mathrm{d}}\left(\mu_{T}, k\right)-2 \bar{y}_{1}^{\mathrm{d}}\left(\mu_{T}, k\right)\right),
\end{aligned}
$$

with

$$
=\frac{c_{1, \mathrm{~s}}\left(\mu_{T}\right)\left(\sigma_{\mu_{T}}^{2}+\omega_{\mu_{T}}^{2}\right) \prod_{\nu_{T} \neq \mu_{T}} e^{2 \sigma_{\nu_{T}} T} \sin ^{2}\left(\omega_{\gamma_{T}} T\right)}{\sum_{\kappa_{T}}\left(c_{1, \mathrm{~s}}^{2}\left(\kappa_{T}\right)\left(\sigma_{\kappa_{T}}^{2}+\omega_{\kappa_{T}}^{2}\right) \prod_{\nu_{T} \neq \kappa_{T}} e^{2 \sigma_{\nu_{T}} T} \sin ^{2}\left(\omega_{\nu_{T}} T\right)\right)} .
$$

The force limitation discussed here can be implemented very efficiently. Only one additional multiplication, one summation, and one binary shift are needed for each vibrational mode (see (20a)), since the more complicated constants $c_{5}\left(\mu_{T}\right)$ have to be calculated only once and the weighting of $\bar{y}_{2}^{\mathrm{d}}\left(\mu_{T}, k\right)$ has to be performed within the recursive system anyway (compare Figure 3 ).

Discrete realizations of the analytical solutions of the MD initial boundary value problems have been derived in this section. For the linear and nonlinear systems, they resulted in stable and accurate simulations of the transversal vibrating string. The drawback of these straight forward discretization approaches of the MD systems in the frequency domains is the high computational complexity of the resulting realizations. Assuming a typical nylon guitar string with $247 \mathrm{~Hz}$ pitch frequency, 59 eigenmodes have to be calculated up to the Nyquist frequency at $22.050 \mathrm{kHz}$. With an average of 3.1 and 4.2 multiplications per output sample (MPOS) per recursive system for the linear and the nonlinear systems, respectively, the total computational cost results for the whole string in 183 MPOS and 248 MPOS. Note that the fractions of the average MPOS result from the assumption that there are only few time instances where an excitation force acts on the string, such that the input weightings of the recursive systems do not have to be calculated at each sample step. Since this is also assumed for the nonlinear slap force, the fractional part in the nonlinear system is higher than in the linear system.

These computational costs are approximately five times higher than those of the most efficient physical modeling method, the DWG [21]. The next section shows that this disadvantage of the FTM can be fixed by using a multirate approach for the simulation of the recursive systems.

\section{DISCRETIZATION WITH A MULTIRATE APPROACH}

The basic idea using a multirate approach to the FTM realization is that the single modes have a very limited bandwidth as long as the damping coefficients $\sigma_{\mu}$ are small. Subdivid- ing the temporal spectrum into different bands that are processed independently of each other, the modes within these bands can be calculated with a sampling rate that is a fraction of the audio rate. Thus, the computational complexity can be reduced with this method. The sidebands generated by this procedure at audio rate are suppressed with a synthesis filter bank when all bands are added up to the output signal. The input signals of the subsampled modes also have to be subsampled. To avoid aliasing, the respective input signals for the modes are obtained by processing the excitation signal $f_{\mathrm{e}}^{\mathrm{d}}(k)$ through an analysis filter bank. This general procedure is shown with solid lines in Figure 4. It shows several modes (RS \# i), each one running at its respective downsampled rate.

This filter bank approach is discussed in detail in the next two sections for the linear as well as for the nonlinear model of the FTM.

\subsection{Discretization of the linear MD model}

For the realization of the structure shown in Figure 4, two major tasks have to be fulfilled [29]:

(1) designing an analysis and a synthesis filter bank that can be realized efficiently,

(2) developing an algorithm that can simulate band changes of single sinusoids to keep the flexibility of the FTM.

\section{Filter bank design}

There are numerous design procedures for filter banks that are mainly specialized to perfect or nearly perfect reconstruction requirements [30]. In the structure shown in Figure 4 there is no need for a perfect reconstruction as in soundprocessing applications, since the sound production mechanism is performed within the single downsampled frequency bands. Therefore, inaccuracies of the interpolation filters can be corrected by additional weightings of the subsampled recursive systems. Linear phase filters with finite impulse responses (FIR) are used for the filter bank due to the variability of the single sinusoids over time. Furthermore, a realvalued generation of the sinusoids in the form of secondorder recursive systems as shown in Figure 2 is preferred to complex-valued first-order recursive systems. This approach avoids on one hand additional real-valued multiplications of complex numbers. On the other hand, the nonlinear slap implementation can be performed in a similar way for the multirate approach, as explained for the audio-rate realization in Section 4.2. A multirate realization of the FTM with complex-valued first-order systems is described in [31].

To fulfill these prerequisites and the requirement of loworder filters for computational efficiency with necessarily flat filter edges, a filter bank with different downsampling factors for different bands has to be designed. A first step is to design a basic filter bank with $P_{\mathrm{ED}}$ equidistant filters, all using the same downsampling factor $r_{\mathrm{ED}}=P_{\mathrm{ED}}$. Due to the flat filter edges, there will be $P_{\mathrm{ED}}-1$ frequency gaps between the single filters that have neither a sufficient passband amplification nor a sufficient stopband attenuation. These gaps are 


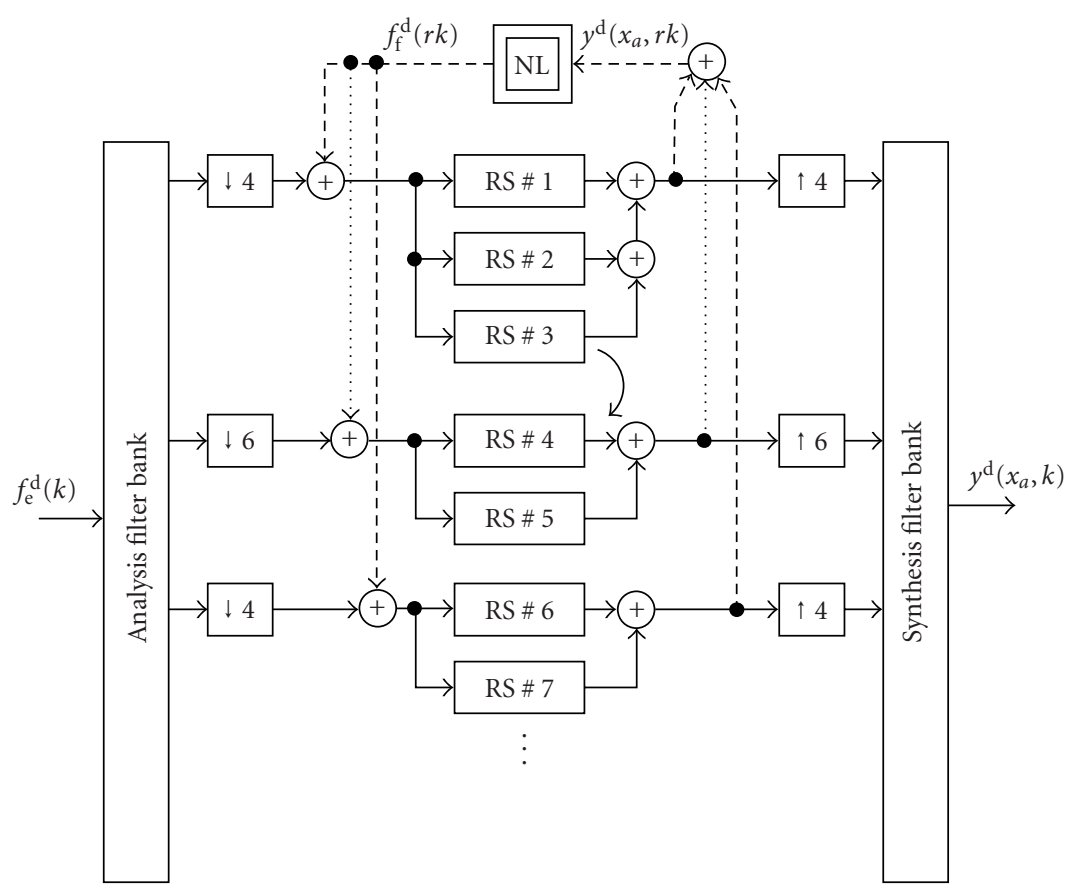

FIGURE 4: Structure of the multirate FTM. Solid lines represent the basic linear system, while dashed and dotted lines represent the extensions for the nonlinear slap force. RS means recursive system. The arrow between RS \# 3 and RS \# 4 indicates a band change.

filled with low-order FIR filters that realize the interpolation of different downsampling factors than $r_{\mathrm{ED}}$. The combination of all filters forms the filter bank. It is used for the analysis and the synthesis filter bank as shown in Figure 4.

An example of this procedure is shown in Figure 5 with $P_{\mathrm{ED}}=4$. The total number of bands is $P=7$. The frequency regions where the single filters are used as passbands in the filter bank are separated by vertical dashed lines. The filters are designed by a weighted least-squares method such that they meet the desired passband bandwidths and stopband attenuations. Note that there are several frequency regions for each filter where the frequency response is not specified explicitly. These so-called "don't care bands" occur since only a part of the Nyquist bandwidth in the downsampled domain is used for the simulation of the modes. Thus, there can only be images of these sinusoids in the upsampled version in distinct regions. All other parts of the spectrum are "don't care bands," for the lowpass filter they are shown as gray areas in Figure 5. Magnitude ripples of $\pm 3 \mathrm{~dB}$ are allowed in the passband which can be compensated by a correction of the weighting factors of the single sinusoids. The stopbands are attenuated by at least $-60 \mathrm{~dB}$, which is sufficient for most hearing conditions. Merely in studio-like hearing conditions larger stopband attenuations must be used such that artifacts produced by using the filter bank cannot be heard.

Due to the different specifications of the filters, concerning bandwidths and edge steepnesses, they have different orders and thus different group delays. To compensate for the different group delays, delay-lines of length $\left(M_{\max }-M_{p}\right) / 2$ are used in conjunction with the filters. The number of coef-

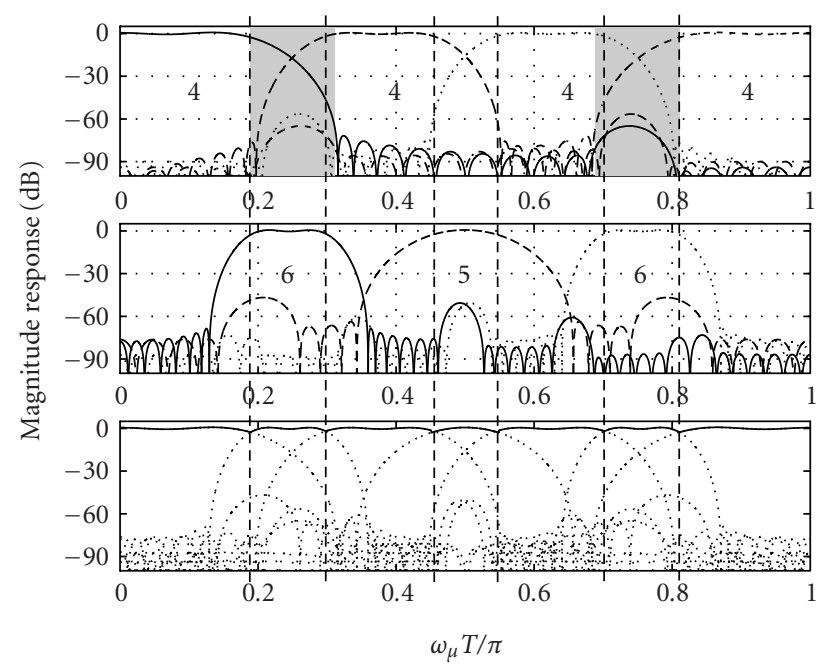

Figure 5: Top: frequency responses of the equidistant filters (with downsampling factor four in this example). Center: frequency responses of the filters with other downsampling factors. Bottom: frequency response of the filter bank. The downsampling factors $r$ are given within the corresponding passbands. The FIR filter orders are between $M_{\min }=34$ and $M_{\max }=72$ in this example. They realize a stopband attenuation of at least $-60 \mathrm{~dB}$ and allow passband ripples of $\pm 3 \mathrm{~dB}$.

ficients of the interpolation filters are denoted by $M_{p}$, where $M_{\max }$ is the maximum order of all filters. The delay lines consume some memory space but no additional computational 
cost [32]. Realizing the filter bank in a polyphase structure, each filter bank results in a computational cost of

$$
C_{\text {filterbank }}=\sum_{p=1}^{P} \frac{M_{p}}{r_{p}} \text { MPOS, }
$$

with the downsampling factors $r_{p}$ of each band. For the example given above, each filter bank needs 73 MPOS. In (21) it is assumed that each band contains at least one mode to be reproduced, so that it is a worst-case scenario. As long as the excitation signal is known in advance, the excitations for each band can be precalculated such that only the synthesis filter bank must be implemented in real time. The case that the excitation signals are known and stored as wavetables in advance is quite frequent in physical modeling algorithms, although the pure physicality of the model is lost by this approach. For example, for string simulations, typical plucking or striking situations can be described by appropriate excitation signals which are determined in advance.

The practical realization of the multirate approach starts with the calculation of the modal frequencies $\omega_{\mu_{T}}$ and their corresponding damping coefficients $\sigma_{\mu_{T}}$. The frequency denotes in which band the mode is synthesized. The coefficients of the recursive systems, as shown in Figure 2 for the audio rate realization, have to be modified in the downsampled domain since the sampling interval $T$ is replaced by

$$
T^{(r)}=r T^{(1)}=r T
$$

Superscript $(r)$ denotes the downsampled simulation with factor $r$. The downsampling factors of the different bands $r_{p}$ are given in the top and center plot of Figure 5. No further adjustments have to be performed for the coefficients of the recursive systems in the multirate approach, since modes can be realized in the downsampled baseband or each of the corresponding images.

\section{Band changes of single modes}

One advantage of the FTM is that the physical parameters of a vibrating object can be varied while playing. This is not only valid for successively played notes but also within one note, as it occurs, for example, in vibrato playing. As far as one or several modes are at the edges of the filter bank bands, these variations can cause the modes to change the bands while they are active. This is shown with an arrow in Figure 4. In such a case, the reproduction cannot be performed by just adjusting the coefficients of the recursive systems with (22) to the new downsampling rate and using the other interpolation filter. This procedure would result in strong transients and in a modification of the modal amplitudes and phases. Therefore, a three-step procedure has to be applied to the band changing modes:

(1) adjusting the internal states of the recursive systems such that no phase shift and no amplitude difference occurs in the upsampled output signal from this mode,

(2) canceling the filter output of the band changing mode,
(3) training of the new interpolation filter to avoid transient behavior.

Similar to the calculation of the instantaneous energy for slap synthesis, also the instantaneous amplitude and phase can be calculated from the internal states of a second-order recursive system, $\bar{y}_{1}$ and $\bar{y}_{2}$. They can be calculated for the old band with downsampling factor $r_{1}$, as well as for the new band with factor $r_{2}$. Demanding the equality of both amplitudes and phases, the internal states of the new band are calculated from the internal states of the old band to

$$
\begin{aligned}
\bar{y}_{1}^{\left(r_{2}\right)}= & \bar{y}_{1}^{\left(r_{1}\right)} \frac{\sin \left(\omega_{\mu} r_{2} T\right)}{\sin \left(\omega_{\mu} r_{1} T\right)} \\
& +\bar{y}_{2}^{\left(r_{1}\right)} e^{\sigma_{\mu} r_{1} T}\left(\cos \left(\omega_{\mu} r_{2} T\right)-\frac{\sin \left(\omega_{\mu} r_{2} T\right)}{\tan \left(\omega_{\mu} r_{1} T\right)}\right), \\
\bar{y}_{2}^{\left(r_{2}\right)}= & \bar{y}_{2}^{\left(r_{1}\right)} e^{\sigma_{\mu}\left(r_{1}-r_{2}\right) T} .
\end{aligned}
$$

The second item of the three-step procedure means that the output of the synthesis interpolation filter must not contain those modes that are leaving that band at time instance $k_{\mathrm{ch}} T$ for time steps $k T \geq k_{\mathrm{ch}} T$. Since the filter bank is a causal system of length $M_{p} T$, the information of the band change must either be given in advance at $\left(k_{\mathrm{ch}}-M_{p}\right) T$ or a turbo filtering procedure has to be applied. In the turbo filtering, the calculations of several sample steps are performed within one sampling interval at the cost of a higher peak computational complexity. In this case, the turbo filtering must calculate the previous outputs of the modes, leaving the band and subtract their contribution to the interpolated output for time instances $k T \geq k_{\mathrm{ch}} T$. Due to the higher peak computational complexity of the turbo filtering and the low orders of the interpolation filters, the additional delay of $M_{p} T$ is preferred here.

In the same way, as the band changing mode must not have an effect on the leaving band from $k_{\mathrm{ch}} T$ on, it must also be included in the interpolation filter of the new band from this time instance on. In other words, the new interpolation filter must be trained to correctly produce the desired mode without transients, as addressed in the third item of the three-step procedure above. It can also be performed with the turbo processing procedure with a higher computational cost or with the delay of $M_{p} T$ between the information of band change and its effect in the output signal.

Now, the linear solution (13) of the transversal vibrating string derived with the FTM is realized also with a multirate approach. Since the single modes are produced at a lower rate than the audio rate, this procedure saves computational cost in comparison to the direct discretization procedure derived in Section 4.1. The amount of computational savings with this procedure is discussed in more detail in Section 6.

\subsection{Extensions for slap synthesis}

In the discretization approach described in Section 4.2 the output $y^{\mathrm{d}}\left(x_{a}, k\right)$ is fed back to the recursive systems via the path of the external force $f_{\mathrm{e}}^{\mathrm{d}}(k)$ (compare Figure 2). Using the same path in the multirate system shown in Figure 4 
would result in a long delay within the feedback path due to the delays in the interpolation filters of the analysis and the synthesis filter bank. Furthermore, the analysis filter bank should not be realized in real time as long as the excitation signal is known in advance.

Fortunately, the recursive systems calculate directly the instantaneous deflection of the single modes, but in the downsampled domain. Considering a system where only modes are simulated in baseband, the signal can be fed back in between the down- and upsampled boxes in Figure 4 and thus directly in the downsampled domain. In comparison to the full-rate system, the observation of the penetration of the string into the fret might be delayed by up to $\left(r_{p}-1\right) T$ seconds. This delay results in a different slap force, but applying the stabilization procedure described in Section 4.3 the stability is guaranteed.

However, in realistic simulations there are also modes in the higher frequency bands than just in the baseband. This modifies the simulations described above in two ways:

(i) the deflection of the string and thus the penetration into the fret depends on the modes of all bands,

(ii) there is an interaction due to nonlinear slap force between all modes in all bands.

The calculation of the instantaneous string deflection in the downsampled rates is rarely possible, since there are various downsampling rates as shown in Figure 4. Thus, there are only a few time instances $k_{\text {all }} T$, where the modal deflections are updated in all bands at the same time. Since in almost all bands one sample value of the recursive systems represents more than half the period of the mode, it is not reasonable to use the previously calculated sample value for the calculation of the deflection at time instances $k T \neq k_{\text {all }} T$. However, all the equidistant bands of the filter bank as shown on top of Figure 5 have the same downsampling factor and can thus represent the same time instances for the calculation of the deflection. Furthermore, most of the energy of guitar string vibrations is in the lower modes [28], such that the deflection is mostly defined by the modes simulated in the lowest bands. Therefore, the string deflection is determined here at each $r_{1}$ th audio sample from all equidistant bands and each $\left(k \bmod r_{1}=0\right) \wedge\left(k \bmod r_{2}=0\right)$ th audio sample from all equidistant bands and bands with the downsampling rate of the lowest band-pass. This is shown in the right dashed and dotted paths in Figure 4. In the example of Figure 5, in each twelfth audio sample the deflection is calculated from the four equidistant bands and each twelfth audio sample it is calculated also from the second and sixth bands.

In the same way the string deflection is calculated with varying participation of the different bands, also the slap force is only applied to modes in these bands as shown in the left dashed and dotted paths in Figure 4. This procedure has two effects: firstly, there is no interaction between all modes at all (downsampled) time instances from the slap force. Secondly, the slap force itself, being an impulse-like signal with a bright spectrum, is filtered by the filter bank. The first effect is not that important since the procedure ensures interactions between most modes but it only restricts them to few time instances, in the example above every fourth or twelfth audio sample. These low delays of the interaction are not noticeable. The second effect can be handled by adding impulses directly to the interpolation filters of the synthesis filter bank. The weights of the impulses in each band are determined by the difference between the sum of all slap force impulses in all bands and the applied slap force impulses in that band. In that way, a slap force, only applied to baseband modes, produces a nearly white noise slap signal at audio rate.

The stabilization procedure described in Section 4.3 can be also applied to the multirate realization of the nonlinear slap force. The only differences to the audio rate simulations are that $T$ is replaced by $r_{p} T$ as given in (22) and the summation for the calculation of the stable slap force $f_{\mathrm{f}}^{\mathrm{d}}(k)$ as given in (20a) is only performed over the modes realized in the participating bands. Thus, there are time instances where the slap force is only applied to the modes in the equidistant bands and time instances where it is applied also to bands with another downsampling factor. This is shown with the dotted lines in Figure 4. Due to the different cases of participating bands, also two versions of the constants $c_{5}\left(\mu_{T}\right)$ have to be calculated, since the products and sums in (20b) depend only on the participating modes.

Now, a stable and realistic simulation of the nonlinear slap force is also obtained in the multirate realization. In the nonlinear case, the simulation accuracy obviously decreases with higher downsampling factors and thus with an increasing number of bands. This effect is discussed in more detail in the next section.

\section{SIMULATION ACCURACY AND COMPUTATIONAL COMPLEXITY}

In the previous sections, stable, linear and nonlinear, discrete FTM models have been derived. In the next sections, the simulation accuracies of these models and their corresponding computational complexities are discussed.

\subsection{Simulation accuracies}

For the linearly vibrating string, the discrete realization of the single modes at full rate is an exactly sampled version of the continuous modes. This is true as long as the input force can be modeled with discrete impulses, since the impulseinvariant transformation is used as explained in Section 4.1. However, the exactness of the complete system is lost with the truncation of the summation of partials in (12) to avoid aliasing effects. Therefore, the results are only accurate as long as the excitation signal has only low energy in the truncated high frequency range. This is true for the guitar and most other musical instruments [28] and, furthermore, the neglected higher partials cannot be received by the human auditory system as long as the sampling interval $T$ is chosen small enough. Since the audible modes are simulated exactly and the simulation error is out of the audible range, the FTM is used here as an optimized discretization approach for sound synthesis applications. 
In multirate simulations of linear systems as described in Section 5.1, the single modes are produced exactly within the downsampled domain. But due to the imperfectness of the analysis filter bank, modes are not only excited by the correct frequency components of the excitation force, but also by aliasing terms that occur with downsampling. In the same way, the images, produced by upsampling the outputs of the recursive systems, are not suppressed perfectly with the synthesis filter bank. However, the filter banks have been designed such that the stopband suppressions are at least $-60 \mathrm{~dB}$. This is sufficient for most listening conditions as defined in Section 5.1. Furthermore, the filters are designed in a least-mean-squares sense such that the energy of the side lobes in the stopbands is minimized. Further filter bank optimizations with respect to the human auditory system are difficult since the filter banks are designed only once for all kinds of mode configurations concerning their positions and amplitude relations in the simulated spectrum.

In the audio rate string model excited nonlinearly with the slap force as described in Section 4.2, the truncation of the infinite sum in (16) also effects the accuracy of the lower modes through the nonlinearity. The simulations are accurate only as long as the external excitation and the nonlinearity have low contributions to the higher modes. Although the external excitation contributes rarely to the higher modes, there is an interaction between all modes due to the slap force. This interaction grows with the modal frequencies. It can be directly seen in the coefficients $c_{5}\left(\mu_{T}\right)$ in $(20 \mathrm{~b})$, since they have larger absolute values for higher frequencies. However, the force contributions of the omitted modes are distributed to the simulated modes since the denominator of (20b) decreases for less simulated partials. Furthermore, the sign of $c_{5}\left(\mu_{T}\right)$ changes with $\mu_{T}$ due to (18) as well as the expression in parenthesis of (20a) does with time. Thus, there is a bidirectional interaction between low and high modes and not only an energy shift from low to high frequencies. $\mathrm{Ne}$ glecting modes out of the audible range results in less energy fluctuations of the audible modes. But since the neglected energy fluctuations have high frequencies, they are also out of the audible range.

In the multirate implementation of the nonlinear model as described in Section 5.2, the interactions between almost all modes are retained. It is more critical here that the observation of the fret-string penetration might be delayed by several audio samples. This circumvents not only the strict limitation of the string deflection by the fret, but is also changes the modal interactions because the nonlinear system is not time-invariant. However, the audible slap effect stays similar to the full-rate simulations and sounds realistic. Audio examples can be found at http://www.LNT.de/ traut/JASP04/sounds.html.

It has been shown that the FTM realizes the continuous solutions of the physical models of the vibrating string accurately. With the multirate approach, the FTM looses the exactness of the linear audio rate model, but the inaccuracies cannot be heard. For the nonlinear model, the multirate approach leads to audible differences compared to the audio rate simulations, but the characteristics of the slap sounds are preserved. Thus, simplifications and computational savings due to the filter bank approach are performed here with respect to the human auditory system.

\subsection{Computational complexities}

The computational complexities of the FTM are explained with two typical examples, a single bass guitar string simulated in different qualities and a six-string acoustic guitar. The first example simulates the vibration of one bass guitar string with fundamental frequency of $41 \mathrm{~Hz}$. The corresponding physical parameters can be found, for example, in [12]. This string is simulated in different sound qualities by varying the number of simulated modes from 1 to 117 , which corresponds to the simulation of all modes up to the Nyquist frequency with a sampling frequency of $f_{\mathrm{s}}=$ $44.1 \mathrm{kHz}$.

Figure 6 shows the dependency of the computational complexities on the number of simulated modes and thus the simulation accuracy or sound quality. The procedure used here to enhance the sound quality consists of simulating more and more modes in consecutive order from the lowest mode on. Thus, the enhancement of the sound quality sounds like opening the lowpass in subtractive synthesis. The upper plot shows the computational complexities for the linear system, simulated at audio rate and with the multirate approach using filter banks with $P=7$ and $P=15$. The bottom plot shows the corresponding graphs for the nonlinear systems. It is assumed that the external forces only act on the string at one tenth of the output samples such that the weighting of the inputs do not have to be performed at each time instance. Thus, each linear recursive system needs 3.1 MPOS for the calculation of one output sample, whereas the nonlinear system needs 4.2 MPOS.

It can be seen that the multirate implementations are much more efficient than the audio-rate simulations, except for simulations with very few modes. With all 117 simulated modes, the relation between audio rate and multirate simulations $(P=7)$ is 363 MPOS to 157 MPOS for the linear system and 492 MPOS to 187 MPOS for the nonlinear system. This is a reduction of the computational complexity of more than $60 \%$.

The steps in the multirate graphs denote the offset of the filter bank realization and that the interpolations of the filter bank bands are only calculated as long as there is at least one mode simulated in those bands. On the one hand, the regions between the steps are steeper in the filter bank with $P=7$ than in that with $P=15$ due to the higher downsampling factors in filter banks with more bands. On the other hand, the steps are higher for filter banks with more bands due to the higher interpolation filter orders. In this example, the multirate approach with $P=7$ is superior to the filter bank with $P=15$ for high qualities, since there are only a few modes simulated in the higher bands of $P=15$, but the filter bank offset is higher. For other configurations with a higher number of simulated modes, this situation is different as shown in the next example. 


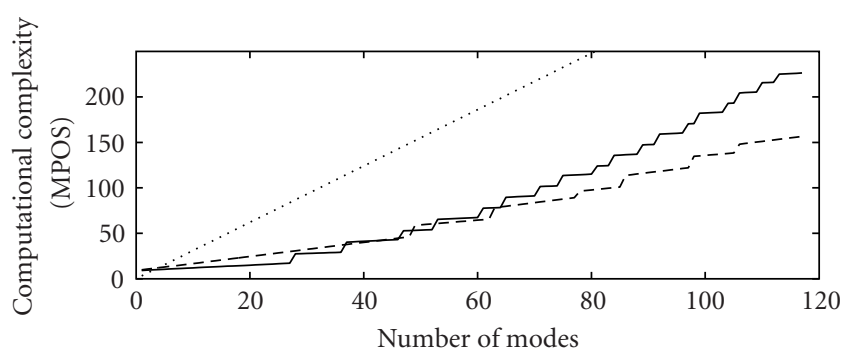

(a)

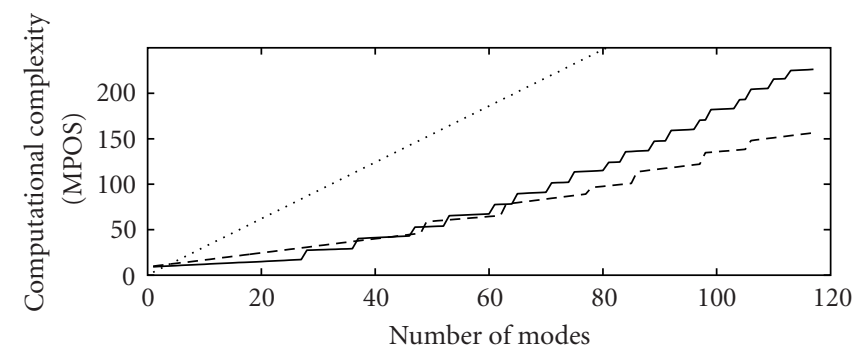

(b)

FIGURE 6: Computational complexities of the FTM simulations dependent on the number of simulated modes at audio rate (dotted line), and with multirate approaches with $P=7$ (dashed line) and $P=15$ (solid line). (a): Linearly vibrating string, (b): vibrating string with nonlinear slap forces.

The second example shows the computational complexities of the simultaneous simulations of six independent strings as they occur in an acoustic guitar. Obviously, there is only one interpolation filter bank needed for all strings. The average number of simulated modes for each guitar string is assumed to be 60. In contrast to the first example, it is assumed that the modes are equally distributed in the frequency domain, such that at least one mode is simulated in each band.

Figure 7 shows that the computational complexities depend on the choice of the used filter bank. On the one hand, each filter bank needs a fixed amount of computational cost which grows with the number of used bands. On the other hand, filter banks with more bands provide higher downsampling factors for the production of the sinusoids which saves computational cost. Thus, the choice of the optimal filter bank depends on the number of simultaneously simulated modes. For practical implementations this has to be estimated in advance.

It can be seen that for the linear case (solid line) the minimum computational cost is 272 MPOS using the filter bank with $P=11$. In the nonlinear case, the filter bank with $P=15$ has the minimum computational cost with 319 MPOS for the simulation of all six strings. Compared to the audio-rate simulations with 1116 MPOS and 1512 MPOS for the linear and nonlinear case, respectively, the multirate simulations allow computational savings up to $79 \%$. Thus, the multirate simulations have a computational complexity of approximately 45 MPOS (53 MPOS) for each linearly (nonlinearly) simulated string.

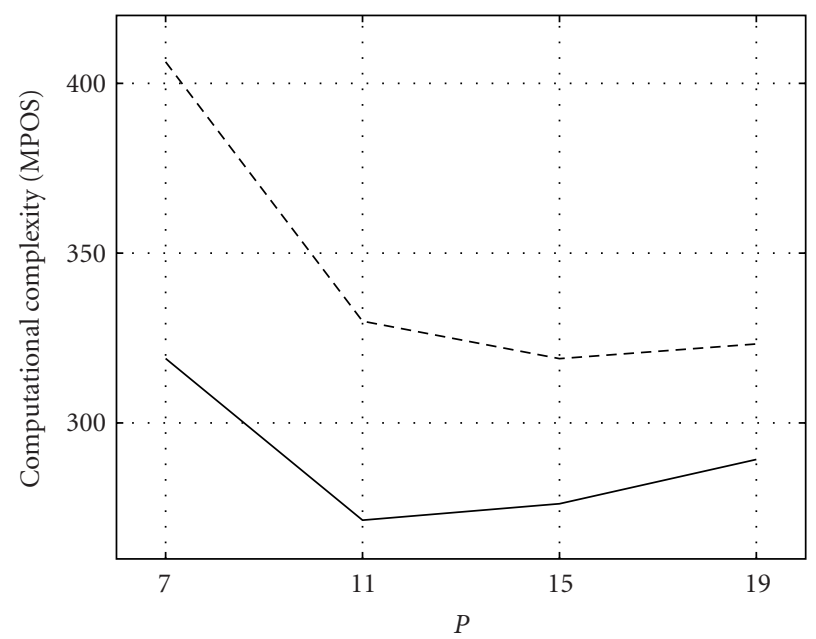

FIGURE 7: Computational complexities of the FTM simulations of a six-string guitar dependent on the number of bands for the multirate approach. Solid line: linearly vibrating string. Dashed line: vibrating string with nonlinear slap forces.

Compared to high quality DWG simulations, the computational complexities of the multirate FTM approach are nearly the same. Linear DWG simulations need up to 40 MPOS for the realization of the reflection filters [21] and the nonlinear limitation of the string by the fret additionally needs 3 MPOS per fret position [22]. 


\section{CONCLUSIONS}

The complete procedure of the FTM has been described from the basic physical analysis of a vibrating structure resulting in an initial boundary value problem via its analytical solution to efficient digital multirate implementations. The transversal vibrating dispersive and lossy string with a nonlinear slap force served as an example. The novel contribution is a thorough investigation of the implementation and the properties of a multirate realization.

It has been shown that the differences between audiorate and multirate simulations for linearly vibrating string simulations are not audible. The differences of the nonlinear simulations were audible but the multirate approach preserves the sound characteristics of the slap sound. The application of the multirate approach saves almost $80 \%$ of the computational cost at audio rate. Thus, it is nearly as efficient as the most popular physical modeling method, the DWG.

The multirate FTM is by far not limited to the example of vibrating strings. It can be used in a similar way to spatially multidimensional systems, like membranes or plates, or even to other physical problems like heat flow or diffusion.

\section{ACKNOWLEDGMENTS}

The authors would like to thank Vesa Välimäki for numerous discussions and his help in the filter bank design for the multirate FTM. Furthermore, the financial support of the Deutsche Forschungsgemeinschaft (DFG) for this research is greatly acknowledged.

\section{REFERENCES}

[1] C. Roads, S. Pope, A. Piccialli, and G. De Poli, Eds., Musical Signal Processing, Swets \& Zeitlinger, Lisse, The Netherlands, 1997.

[2] L. Hiller and P. Ruiz, "Synthesizing musical sounds by solving the wave equation for vibrating objects: Part I," Journal of the Audio Engineering Society, vol. 19, no. 6, pp. 462-470, 1971.

[3] A. Chaigne and V. Doutaut, "Numerical simulations of xylophones. I. Time-domain modeling of the vibrating bars," Journal of the Acoustical Society of America, vol. 101, no. 1, pp. 539-557, 1997.

[4] A. Chaigne, "On the use of finite differences for musical synthesis. Application to plucked stringed instruments," Journal d'Acoustique, vol. 5, no. 2, pp. 181-211, 1992.

[5] A. Chaigne and A. Askenfelt, "Numerical simulations of piano strings. I. A physical model for a struck string using finite difference methods," Journal of the Acoustical Society of America, vol. 95, no. 2, pp. 1112-1118, 1994.

[6] M. Karjalainen, "1-D digital waveguide modeling for improved sound synthesis," in Proc. IEEE Int. Conf. Acoustics, Speech, Signal Processing, vol. 2, pp. 1869-1872, IEEE Signal Processing Society, Orlando, Fla, USA, May 2002.

[7] C. Erkut and M. Karjalainen, "Finite difference method vs. digital waveguide method in string instrument modeling and synthesis," in Proc. International Symposium on Musical Acoustics, Mexico City, Mexico, December 2002.

[8] C. Cadoz, A. Luciani, and J. Florens, "Responsive input devices and sound synthesis by simulation of instrumental mechanisms: the CORDIS system," Computer Music Journal, vol. 8, no. 3, pp. 60-73, 1984.
[9] J. M. Adrien, "Dynamic modeling of vibrating structures for sound synthesis, modal synthesis," in Proc. AES 7th International Conference, pp. 291-299, Audio Engineering Society, Toronto, Canada, May 1989.

[10] G. De Poli, A. Piccialli, and C. Roads, Eds., Representations of Musical Signals, MIT Press, Cambridge, Mass, USA, 1991.

[11] G. Eckel, F. Iovino, and R. Caussé, "Sound synthesis by physical modelling with Modalys," in Proc. International Symposium on Musical Acoustics, pp. 479-482, Le Normant, Dourdan, France, July 1995.

[12] L. Trautmann and R. Rabenstein, Digital Sound Synthesis by Physical Modeling Using the Functional Transformation Method, Kluwer Academic Publishers, New York, NY, USA, 2003.

[13] D. A. Jaffe and J. O. Smith, "Extensions of the Karplus-Strong plucked-string algorithm," Computer Music Journal, vol. 7, no. 2, pp. 56-69, 1983.

[14] K. Karplus and A. Strong, "Digital synthesis of plucked-string and drum timbres," Computer Music Journal, vol. 7, no. 2, pp. 43-55, 1983.

[15] J. O. Smith, "Physical modeling using digital waveguides," Computer Music Journal, vol. 16, no. 4, pp. 74-91, 1992.

[16] J. O. Smith, "Efficient synthesis of stringed musical instruments," in Proc. International Computer Music Conference, pp. 64-71, Tokyo, Japan, September 1993.

[17] M. Karjalainen, V. Välimäki, and Z. Jánosy, "Towards highquality sound synthesis of the guitar and string instruments," in Proc. International Computer Music Conference, pp. 56-63, Tokyo, Japan, September 1993.

[18] M. Karjalainen, V. Välimäki, and T. Tolonen, "Plucked-string models, from the Karplus-Strong algorithm to digital waveguides and beyond," Computer Music Journal, vol. 22, no. 3, pp. 17-32, 1998.

[19] R. Rabenstein, "Discrete simulation of dynamical boundary value problems," in Proc. EUROSIM Simulation Congress, pp. 177-182, Vienna, Austria, September 1995.

[20] L. Trautmann and R. Rabenstein, "Digital sound synthesis based on transfer function models," in Proc. IEEE Workshop on Applications of Signal Processing to Audio and Acoustics, pp. 83-86, IEEE Signal Processing Society, New Paltz, NY, USA, October 1999.

[21] L. Trautmann, B. Bank, V. Välimäki, and R. Rabenstein, "Combining digital waveguide and functional transformation methods for physical modeling of musical instruments," in Proc. Audio Engineering Society 22nd International Conference on Virtual, Synthetic and Entertainment Audio, pp. 307-316, Espoo, Finland, June 2002.

[22] E. Rank and G. Kubin, "A waveguide model for slapbass synthesis," in Proc. IEEE Int. Conf. Acoustics, Speech, Signal Processing, pp. 443-446, IEEE Signal Processing Society, Munich, Germany, April 1997.

[23] M. Kahrs and K. Brandenburg, Eds., Applications of Digital Signal Processing to Audio and Acoustics, Kluwer Academic Publishers, Boston, Mass, USA, 1998.

[24] L. Trautmann and R. Rabenstein, "Stable systems for nonlinear discrete sound synthesis with the functional transformation method," in Proc. IEEE Int. Conf. Acoustics, Speech, Signal Processing, vol. 2, pp. 1861-1864, IEEE Signal Processing Society, Orlando, Fla, USA, May 2002.

[25] B. Girod, R. Rabenstein, and A. Stenger, Signals and Systems, John Wiley \& Sons, Chichester, West Sussex, UK, 2001.

[26] R. V. Churchill, Operational Mathematics, McGraw-Hill, New York, NY, USA, 3rd edition, 1972.

[27] R. Rabenstein and L. Trautmann, "Digital sound synthesis of string instruments with the functional transformation 
method," Signal Processing, vol. 83, no. 8, pp. 1673-1688, 2003.

[28] N. H. Fletcher and T. D. Rossing, The Physics of Musical Instruments, Springer-Verlag, New York, NY, USA, 1998.

[29] L. Trautmann and V. Välimäki, "A multirate approach to physical modeling synthesis using the functional transformation method," in Proc. IEEE Workshop on Applications of Signal Processing to Audio and Acoustics, pp. 221-224, IEEE Signal Processing Society, New Paltz, NY, USA, October 2003.

[30] P. P. Vaidyanathan, Multirate Systems and Filter Banks, Prentice Hall, Englewood Cliffs, NJ, USA, 1993.

[31] S. Petrausch and R. Rabenstein, "Sound synthesis by physical modeling using the functional transformation method: Efficient implementation with polyphase filterbanks," in Proc. International Conference on Digital Audio Effects, London, UK, September 2003.

[32] B. Bank, "Accurate and efficient method for modeling beating and two-stage decay in string instrument synthesis," in Proc. MOSART Workshop on Current Research Directions in Computer Music, pp. 134-137, Barcelona, Spain, November 2001.

L. Trautmann received his "Diplom-Ingenieur" and "Doktor-Ingenieur" degrees in electrical engineering from the University of Erlangen-Nuremberg, in 1998 and 2002, respectively. In 2003 he was working as a Postdoc in the Laboratory of Acoustics and Audio Signal Processing at the Helsinki University of Technology, Finland. His research interests are in the simulation of multidimensional systems with focus on digital

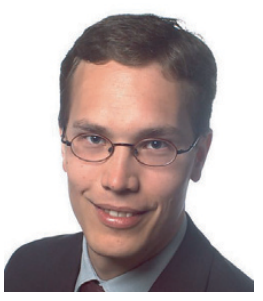
sound synthesis using physical models. Since 1999, he published more than 25 scientific papers, book chapters, and books. He is a holder of several patents on digital sound synthesis.

R. Rabenstein received his "Diplom-Ingenieur" and "Doktor-Ingenieur" degrees in electrical engineering from the University of Erlangen-Nuremberg, in 1981 and 1991, respectively, as well as the "Habilitation" in signal processing in 1996. He worked with the Telecommunications Laboratory of this university from 1981 to 1987 and since 1991. From 1998 to 1991, he was with the Physics Department of the University of

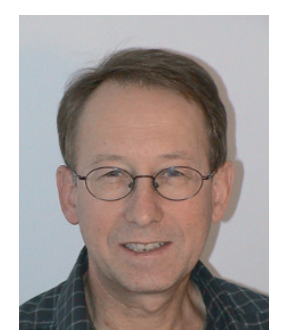
Siegen, Germany. His research interests are in the fields of multidimensional systems theory and simulation, multimedia signal processing, and computer music. He serves in the IEEE TC on Signal Processing Education. He is a Board Member of the School of Engineering of the Virtual University of Bavaria and has participated in several national and international research cooperations. 